\title{
Regarding the Optical Properties of Porous Layers Prepared on Si Substrates
}

\author{
Emil Pinčík ${ }^{1}$, Robert Brunner ${ }^{1}$, Hikaru Kobayashi ${ }^{2}$, Pavel Vojtek ${ }^{3}$, Zuzana Zábudlá ${ }^{3}$, Milan Mikula ${ }^{4}$, Jan Greguš $\breve{s}^{3}$ \\ and Michal Kučera ${ }^{5}$ \\ 1. Institute of Physics, SAS, Dubravska cesta 9, Bratislava 84511, Slovak Republic \\ 2. Institute of Scientific and Industrial Research, Osaka University, Mihogaoka, Ibaraki, Osaka 567-0047, Japan \\ 3. FMPI of Comenius University, Mlynská dolina F2, Bratislava 84248, Slovak Republic \\ 4. Faculty of Chemical and Food Technology of STU, Radlinského 9, Bratislava 81237, Slovak Republic \\ 5. Institute of Electrical Engineering SAS, Dubravska cesta 9, Bratislava 84104, Slovak Republic
}

Received: October 19, 2017 / Accepted: October 30, 2017 / Published: November 30, 2017

\begin{abstract}
The paper deals with the complex refractive index and photoluminescence in the IR-VIS light region of two sample types (i) black p-type silicon (BSi) produced by the surface structure chemical transfer method using Pt catalytic mesh, and (ii) porous p-type silicon prepared by standard electrochemical etching. We present, compare, and discuss the values of the IR-VIS complex refractive index obtained by calculation using the Kramers-Kronig transformation and the photoluminescence properties thereof. The results indicate that differences between the optical properties of the BSi and the porous Si are given by (a) the oxidation procedure of $\mathrm{BSi}$, (b) the thickness of the formed black and porous Si layer, and by (c) the porosity of both layer types. We assume that the photoluminescence signal generated by oxidized BSi structures can be mainly related to the quantum confinement effect, while the photoluminescence of the porous p-type $\mathrm{Si}$ is caused by the optical activity of the $\mathrm{SiO}_{\mathrm{x}} \mathrm{H}_{\mathrm{y}}$ compounds covering its surface region.
\end{abstract}

Key words: Black silicon, porous silicon, complex refractive index, photoluminescence.

\section{Introduction}

Black silicon structures are currently used in the development of new-type Si-based solar cells that do not have anti-reflection coatings. The anti-reflection properties of black silicon structures enable the formation of solar cells with conversion efficiencies of over $19 \%$ on large areas. Such efficiency of corresponding solar cells can be increased by the suitable passivation of defects created during the etching procedure. The oxidation of prepared black silicon structures is one of the most suitable passivation procedures. Si nano-crystallites covered by $\mathrm{SiO}_{(x)}$ create one of the basic elements of BSi structures used

Corresponding author: Emil Pinčík, Ph.D., research fields: research of passivated structures of standard porous silicon and black silicon. for the development of corresponding high-efficiency solar cells.

The SSCT (surface-structure chemical transfer) method can produce a nano-crystalline Si black color layer on c-Si with a thickness range of $\sim 50 \mathrm{~nm}$ to $\sim 300$ $\mathrm{nm}$ by the contact of c-Si immersed in $\mathrm{HF}+\mathrm{H}_{2} \mathrm{O}_{2}$ chemical solutions with a catalytic mesh.

Formation and properties of porous Si structures are reviewed in Ref. [1]. The second type of porous $\mathrm{Si}$ structures was formed by the standard electrochemical manner in a solution of HF and methanol under the influence of the electric field between the Si sample (+) and Pt electrode (-).

Researchers at ISIR Osaka University have developed a fabrication method of low reflectivity $\mathrm{Si}$ surfaces in which a mold with catalytic $\mathrm{Pt}$ layer is contacted with Si-immersed $\mathrm{H}_{2} \mathrm{O}_{2}+\mathrm{HF}$ solution. The 
PL maximum of a black silicon layer prepared on polycrystalline $\mathrm{Si}$ wafer at room temperature had the position $\sim 1.85 \mathrm{eV}$ [2]. A similar method was also applied for the preparation of the BSi samples in this contribution. An additional alternative technological approach was developed in the same Japanese laboratories by M. Takahashi et al. [3].

In the contribution [4] we presented an analysis of the photoluminescence properties of as prepared thin ( $\sim 100 \mathrm{~nm}$ thick) multicolor silicon structures.

In this paper we will present, discuss and compare values of the calculated complex refractive index and the photoluminescence properties of oxidized p-type BSi structures and porous p-type $\mathrm{Si}$.

\section{Experiment}

2.1 Preparation of Samples, Measurements and Evaluation

The p-type (100) Si has been treated using Pt mash in the solution $15 \mathrm{wt} \% \mathrm{HF}+25 \mathrm{wt} \% \mathrm{H}_{2} \mathrm{O}_{2}$, and produced with the help of $\mathrm{Pt}$ mash. After the formation of a black layer, the structures were dried and oxidized at $\sim 900{ }^{\circ} \mathrm{C}$. A set of the following three samples was prepared: (a) reference sample, (b) $\mathrm{Si}$ treated by etching solution for 20 seconds, and (c) $\mathrm{Si}$ treated by etching solution for 240 seconds.

The standard porous $\mathrm{Si}$ was prepared in an electrochemical manner using a solution of $\mathrm{HF}$ and $\mathrm{MeOH}$. Electrical voltage $(6-11 \mathrm{~V})$ was applied between the Si sample (positive potential) and the $\mathrm{Pt}$ negative electrode localized in the solution outside the Si sample. A constant current of $30 \mathrm{~mA} \cdot \mathrm{cm}^{-2}$ was maintained during the etching. The following two samples were prepared: (i) with etching time of 1 minute, and (ii) with etching time of 10 minutes.

For the calculation of the complex refractive index of structures, we used IR reflectivity measurements under the angle $30^{\circ}$ and recorded by a Digilab Excalibur FTS 3000 MX spectrometer with the following FTIR spectroscopy software: Resolutions Pro 5.2.0, Agilent with Kramers-Krönig transformation. A PIKE Technologies Specular Reflectance Accessory was used at an angle of $30^{\circ}$. The diameter of the measured area was $10 \mathrm{~mm}$, used resolution: $4 \mathrm{~cm}^{-1}$, number of scans: 60 .

Real and imaginary parts of the complex refractive index can be expressed from the following equations:

$$
\begin{aligned}
& n=\frac{1-r^{2}}{1+r^{2}-2 r \cos \theta} \\
& k=\frac{-2 r \sin \theta}{1+r^{2}-2 r \cos \theta}
\end{aligned}
$$

where $n$ and $k$ are the real and imaginary parts of the refractive index $\hat{n}=n-i \cdot k, R=r^{2}$ is the experimentally-measured reflectance, and $\theta$ is the phase difference between the incident and reflected waves. This can be calculated as Ref. [5]:

$$
\theta_{c}=2 \frac{\omega_{c}}{\pi} \int_{0}^{\infty} \frac{\ln r(\omega)-\ln r\left(\omega_{c}\right)}{\omega^{2}-\omega_{c}^{2}} d \omega
$$

where $\theta_{c}$ is the quantity $\theta$ for the given $\omega_{c}, \omega_{c}$ and $\omega$ are the frequencies.

For the measurements of the photoluminescence properties of the $\mathrm{BSi}$ and porous Si structures, we used the same equipment as published in Ref. [4].

\subsection{Experimental Results and Discussion}

Fig. 1 illustrates the IR dispersion characteristics of the refractive index $n$ of reference $\mathrm{Si}$, and two oxidized BSi structures prepared with Pt catalytic mesh. We assume that in both records of oxidized BSi structures in the region $500-1,200 \mathrm{~cm}^{-1}$, the increased absorbance of structures caused by $\mathrm{Si}-\mathrm{O}$ and $\mathrm{Si}-\mathrm{OH}$ bonds modifies the calculated values of refractive index $n$.

Fig. 2 illustrates the corresponding IR dispersion characteristics of extinction coefficients $k$ of all three samples presented in Fig. 1

Fig. 3 shows the photoluminescence signal obtained from the oxidized BSi sample at room temperature and at $6 \mathrm{~K}$.

Fig. 4 presents the photoluminescence signal measured on p-type porous Si structure at $40 \mathrm{~K}$. 


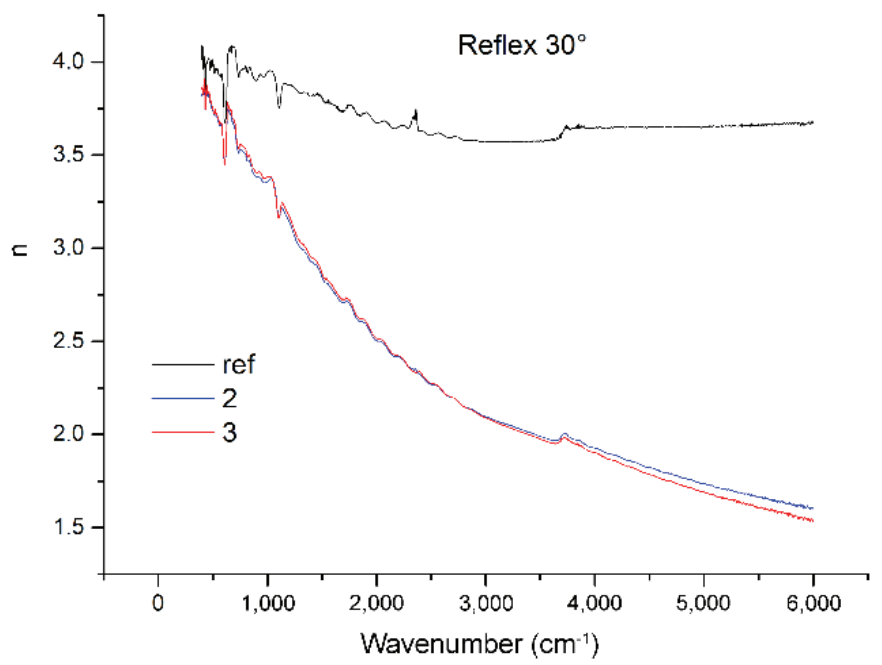

Fig. 1 IR dispersion characteristics of refractive index $n$ of reference $\mathrm{Si}$ (ref) and two BSi structures $(2,3)$.

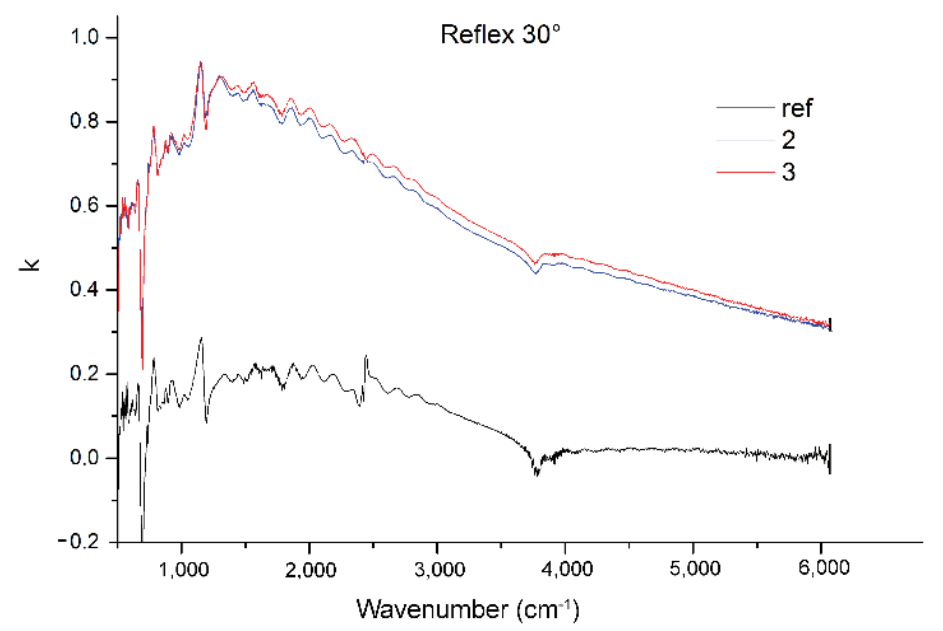

Fig. 2 IR dispersion characteristics of extinction coefficient $k$ of reference Si (ref) and two BSi structures $(2,3)$.

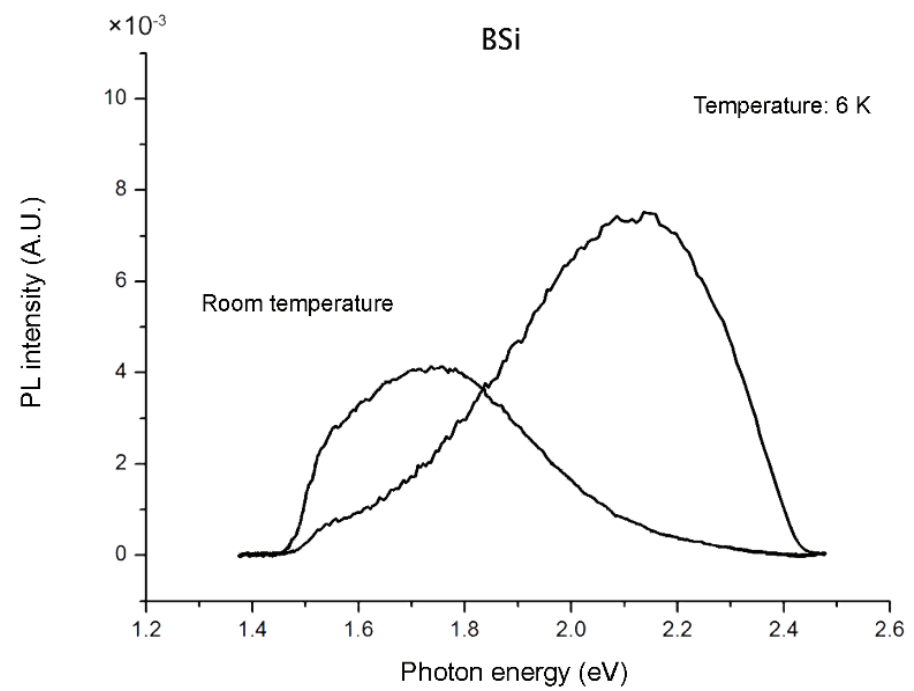

Fig. 3 Photoluminescence signal obtained from the oxidized BSi sample at room temperature and at $6 \mathrm{~K}$. 


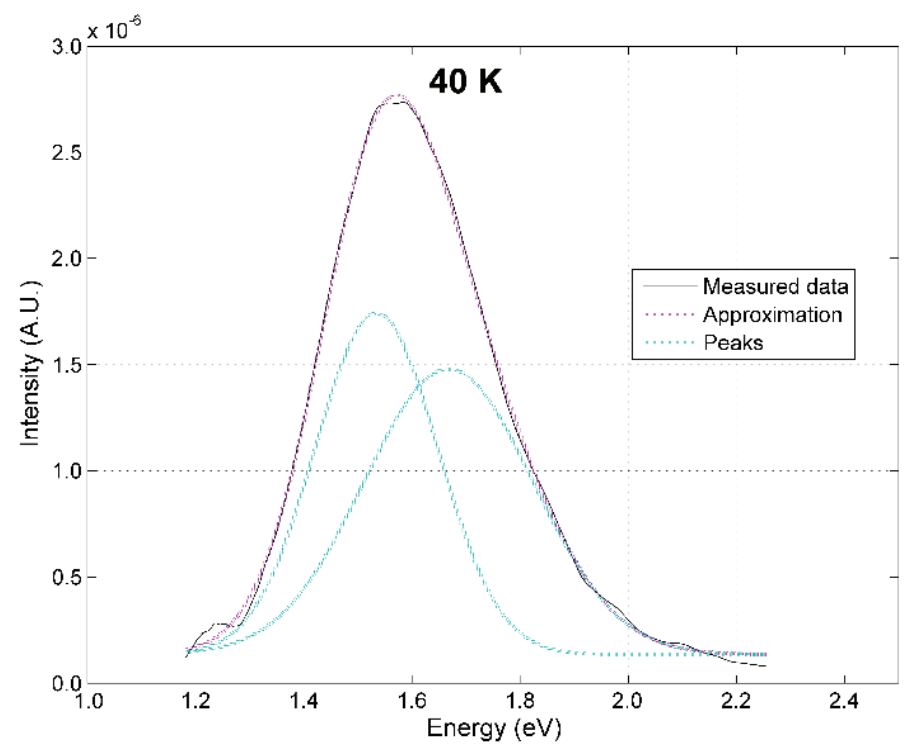

Fig. 4 Photoluminescence signal measured on p-type porous Si structure at $40 \mathrm{~K}$.

Table 1 Positions of maxima of photoluminescence signals presented in Figs. 3 and 4.

\begin{tabular}{lllll}
\hline No. & BSi $(\mathrm{eV}) 6 \mathrm{~K}$ & BSi $(\mathrm{eV}) \mathrm{RT}$ & $\Delta$ Energy BSi $(\mathrm{eV})$ & Porous $\mathrm{Si}(\mathrm{eV})$ \\
\hline 1 & 1.86 & 1.57 & 0.29 & 1.53 \\
2 & 2.09 & 1.71 & 0.38 & 1.67 \\
3 & 2.24 & 1.88 & 0.36 & - \\
\hline
\end{tabular}

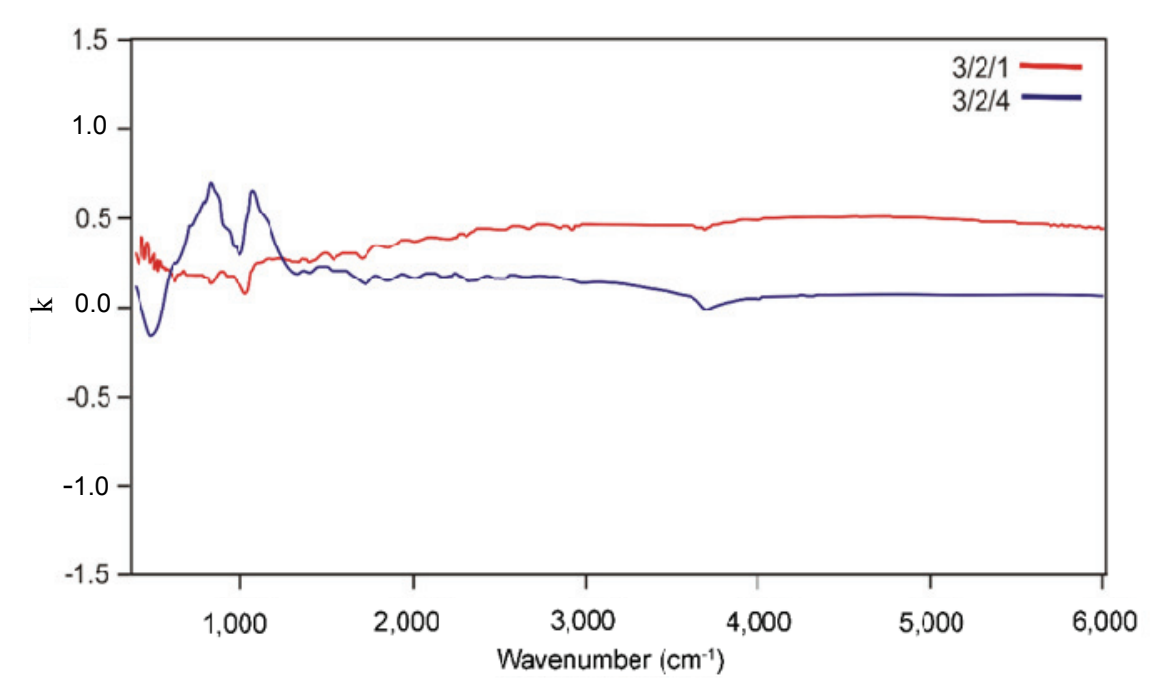

Fig. 5 IR dispersion dependences of extinction coefficient $k$ of p-type porous Si structures prepared with etching time: (a) 1 minute (3/2/1), (b) 10 minutes (3/2/4).

Table 1 shows the positions of maxima of photoluminescence signals presented in Figs. 3 and 4. They were determined by the fitting procedure.

Figs. 5 and 6 show the IR dispersion dependences of extinction coefficient $k$ and refractive index $n$, respectively, of two porous p/type $\mathrm{Si}$ structures prepared with etching time: (i) 1 minute $(3 / 2 / 1)$, (ii) 10 minutes $(3 / 2 / 4)$.

The results indicate that small differences between the optical properties of thermally-oxidized BSi and standard p-type porous $\mathrm{Si}$ is given by the oxidation procedure of BSi, the thickness of the formed black and porous Si layer, and the porosity of both types of formed layers. The utilization of catalytic metal in BSi 


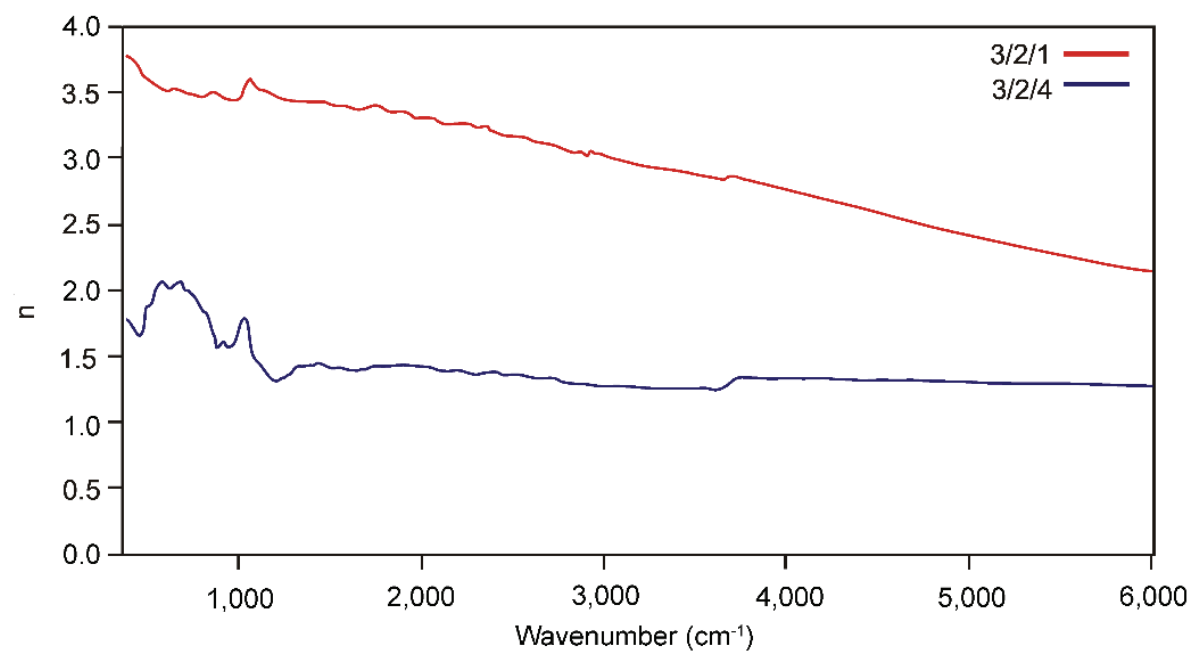

Fig. 6 IR dispersion dependences of refractive index $\boldsymbol{n}$ of p-type porous Si structures prepared with etching time: (a) 1 minute (3/2/1), (b) 10 minutes (3/2/4).

does not strongly influence the observed IR optical properties in comparison with standard porous Si. The optical properties of the reference Si (see Fig. 1) are influenced by the surface native oxide, and/or by the mechanically-damaged surface region of crystalline $\mathrm{Si}$ wafers. We assume that the photoluminescence of the BSi structures (Fig. 3) is related to the quantum confinement effect. A blue shift of the photoluminescence signal of standard porous $\mathrm{Si}$ (see Fig. 4, Table 1, and Ref. [6]) with decreasing temperature was not observed. We relate the generation of the photoluminescence signal of standard p-type $\mathrm{Si}$ to the $\mathrm{SiO}_{\mathrm{x}} \mathrm{H}_{\mathrm{y}}$ compounds covering the structure after its formation and drying.

\section{Conclusions}

This contribution deals with the complex refractive index in the IR light region of two sample types (a) thermally-oxidized black p-type silicon (BSi) nano-crystalline specimens produced both by the surface structure chemical transfer method, and (b) standard porous p-type Si.

We present and compare (i) the values of the IR complex refractive index obtained by calculation using the Kramers-Kronig transformation, and (ii) their photoluminescence properties. Small differences were observed in the values of the complex refractive index between thermally oxidized BSi and standard p-type porous Si when they have similar porosity. On the other hand, the photoluminescence signal generated by oxidized $\mathrm{BSi}$ structures is related to the quantum confinement effect, while the photoluminescence of porous p-type $\mathrm{Si}$ is caused - most probably-by the $\mathrm{SiO}_{\mathrm{x}} \mathrm{H}_{\mathrm{y}}$ compounds covering its surface region.

\section{Acknowledgements}

The following APVV-project No.0152/15 and VEGA — projects No.2/0076/15, 1/0900/16, 1/0676/17 agencies partly financially supported this contribution. We would like to thank Prof. K. Imamura of ISIR Osaka University, Japan, Mrs. S. Bacova, and Mr. Peter Zitto (both of the IoP SAS Bratislava) for the preparation of samples and very insightful discussions. We also thank Ms. Julia Tomekova of FMPI Comenius University, Bratislava, Slovakia for her help with this contribution.

\section{References}

[1] Porous Silicon-From Formation to Application. 2016. CDC Press, Taylor and Francis Group, Boca Raton, London, New York, 2016, number of pages: 423.

[2] Imamura, K., JR., F. C. F., Matsumoto, T., and Kobayashi, H. 2013. "Ultra-low Reflectivity Polycrystalline Silicon Surfaces Formed by Surface Structure Chemical Transfer Method." Appl. Phys. Lett. 103 (1): 1195-B15. doi:10.1063/1.4813089. 
[3] Takahashi, M., Fukushima, T., Seino, Y., Kim, W.-B., Imamura, K., and Kobayashi, H. 2013. "Surface Structure Chemical Transfer Method for Formation of Ultralow Reflectivity Si Surfaces." J. Electrochem. Soc. 160 (8): H443. doi:10.1149/2.044308jes.

[4] Pincik, E., Brunner, R., Kobayashi, H., Mikula, M., Vojtek, P., Greguš, J., Zabudla, Z., Imamura, K., and Švec Jr, P. "The Photoluminescence of Multicolor Silicon." Journal of the Chinese Advanced Materials Society 4 (2): 152-71. http://dx.doi.org/10.1080/22243682.2016.1151374.

[5] Palik, E. D. (Ed.) 1985. Handbook of Optical Constants of Solids. Orlando: Academic Press Inc.

[6] Brunner, R., Pinčík, E., Kučera, M., Vojtek, P., and Zábudlá, Z. 2017. "The Temperature Dependence of Photoluminescence Peaks of Porous Silicon Structures." In Proceedings of the 23rd International Conference APCOM 2017, June 12-14, Štrbské Pleso, Slovakia; Editors: J. Vajda, I. Jamnický, p. 207. 